\title{
A model of cavitation for the treatment of a moving liquid metal volume
}

G. S. Bruno Lebon ${ }^{1}$, Koulis Pericleous ${ }^{1}$, Iakovos Tzanakis ${ }^{2,3}$, Dmitry Eskin ${ }^{2,4}$

${ }^{1}$ Computational Science and Engineering Group, University of Greenwich, London, SE10 9LS, United Kingdom

${ }^{2}$ Brunel Centre for Advanced Solidification Technology (BCAST), Brunel University, Uxbridge, Middlesex, UB8 3PH, United Kingdom

${ }^{3}$ Faculty of Technology, Design and Environment, Oxford Brookes University, Wheatley

Campus, Wheatley OX33 1HX, United Kingdom

${ }^{4}$ Smart Materials and Technologies Institute (SMTI), Tomsk State University, Tomsk, 634050, Russia

Corresponding author: G.S.B.Lebon@gre.ac.uk

A homogeneous cavitation model, derived from the Keller-Miksis equation, is developed and applied to the two-phase problem of bubble growth, breakup and propagation in the melt. Numerical simulations of the ultrasonic field emanating from an immersed sonotrode are performed and the calculated acoustic pressure is applied to the source term of the bubble transport equation to predict the generation, propagation and collapse of cavitation bubbles in the melt. The use of baffles to modify the flow pattern and amplify sound waves in a launder conduit is examined to determine the optimum configuration that maximizes the residence time of the liquid in high cavitation activity regions. The simulation results demonstrate that dimensions that match integer wavelengths, and are therefore in resonance with the travelling waves, are desirable since they lead to an increase in the concentration of nucleating bubbles in the liquid compared with other dimensions.

Keywords: Cavitation modelling, Ultrasonics, Liquid aluminium, Homogeneous cavitation, Ultrasonic melt treatment

\section{Introduction}

The treatment of melt with ultrasound has been demonstrated to result in significant improvements in the quality and properties of metallic materials ${ }^{1-4}$ : the beneficial effects of the 
treatment include degassing of dissolved gases, improved wetting, activating inclusions by cleaning the solid-liquid interface, enhancing nucleation and refining the grain structure of the solidified sample ${ }^{1}$. These improvements are attributed to ultrasonic cavitation ${ }^{5}$ : the creation, growth, pulsation and collapse of bubbles in the liquid ${ }^{6,7}$. However, treating large volumes of liquid metal, as is required by processes such as continuous casting, is still elusive; this technology has not yet been successfully transferred to the industry as a result. A fundamental study of the ultrasonic treatment of melt is required to circumvent these difficulties ${ }^{8}$.

Transport equation-based cavitation models applied to the Navier-Stokes equations are commonly encountered in the literature, for example ${ }^{9,10}$. Most of these models have been developed to predict cavitation bubble concentration in water, rather than in liquid metals where evaporation and condensation are not significant. The model of Merkle et al. ${ }^{11,12}$ uses evaporation and condensation terms that are both functions of pressure. Kunz et al. ${ }^{13}$ uses an evaporation term that is a function of pressure, whilst their condensation term is a function of volume fraction.

While both models yield satisfying predictions at different cavitation numbers ${ }^{9}$, the full cavitation models of Athavale et al. ${ }^{14,15}$ and Sauer and Schnerr ${ }^{16}$ are more commonly encountered in the literature. These models enable multidimensional simulation of cavitating flows, the modelling of which is crucial to the design of many engineering devices. In the approach of Athavale et al. ${ }^{14,15}$, the source terms for the bubble mass fraction transport equation are derived from the Rayleigh-Plesset equation ${ }^{17,18}$, which governs the evolution of a spherical bubble ${ }^{19,20}$, to predict the formation and collapse of bubbles in cavitating flows. This model has been used in a metallurgical context by Nastac ${ }^{21}$ for the modelling of solidification structure evolution in a crucible.

In this paper, the full cavitation model of Athavale et al. ${ }^{14,15}$ is modified to be applied in cases involving strong acoustic pressures, for example to determine the bubble concentration in a 
launder conduit. The Keller-Miksis equation, which is more appropriate than the RayleighPlesset equation for these large forcing amplitudes ${ }^{22}$, is used to derive the source terms for the vapour transport equation. An additional turbulence source term, also derived from the KellerMiksis equation, accounts for the increased turbulence created by bubbles as they pulsate and cavitate. These changes are proposed to improve the understanding of the effect of ultrasonic treatment on flowing melt, paving the way to the ultrasonic treatment of liquid metal as a continuous process.

\section{Theory}

\section{Governing Equations}

\section{Wave equations}

The wave equation is usually expressed as

$$
\frac{\partial^{2} p}{\partial t^{2}}-c^{2} \frac{\partial}{\partial x_{i}}\left(\frac{\partial p}{\partial x_{i}}\right)=c^{2} S_{p}
$$

where $p$ is acoustic pressure, $c$ is the speed of sound of the travelling medium and $S_{p}$ are the wave source terms. In this paper, the wave equation is expressed in terms of the continuity and momentum conservation equations.

$$
\begin{aligned}
& \frac{\partial \rho}{\partial t}+\nabla \cdot\left(\rho \boldsymbol{u}^{\prime}\right)=S_{\text {mass }} \\
& \frac{\partial\left(\rho u_{i}{ }^{\prime}\right)}{\partial t}+\nabla \cdot\left(\rho \boldsymbol{u}^{\prime} u_{i}{ }^{\prime}\right)=S_{u_{i}{ }^{\prime}}
\end{aligned}
$$

where $\rho$ is the fluid density and $\boldsymbol{u}^{\prime}$ is the perturbation velocity. The advantage of this formulation lies in the possibility of extending the discretization method of the wave equation to 
higher orders in space and time using fully staggered scheme to resolve acoustic wave propagation more accurately: a summary of this approach is presented elsewhere ${ }^{23}$.

The bubbly fluid density is related to the vapour and non-condensable gas mass fractions, $f_{v}$ and $f_{g}$, according to:

$$
\frac{1}{\rho}=\frac{f_{v}}{\rho_{v}}+\frac{f_{g}}{\rho_{g}}+\frac{1-f_{v}-f_{g}}{\rho_{l}}
$$

where $\rho_{v}$ is the bubble vapour density and $\rho_{g}$ is the non-condensable gas density ${ }^{14}$.

Vapour is negligible when considering liquid aluminium, since the vapour pressure of aluminium at its melting point is $0.000012 \mathrm{~Pa}^{24}$. Aluminium vapour bubbles are therefore unlikely to be formed in the bulk ${ }^{25}$. However, vapour is readily present in water.

The source term $S_{\text {mass }}$ contains mass sources such as vibrating solid surfaces. The forcing terms $S_{u_{i}}{ }^{\prime}$ contain velocity sources due to the vibrating solid surfaces. The speed of sound is dependent on the local density $\rho$ and bulk modulus $\mathrm{K}$ :

$$
c=\sqrt{\mathrm{K} / \rho}
$$

\section{Bubble evolution equation}

The Keller-Miksis equation ${ }^{22}$ governs the evolution of bubble radii:

$$
\left(1-\frac{\dot{R}}{c_{l}}\right) R \ddot{R}+\frac{3}{2}\left(1-\frac{1}{3} \frac{\dot{R}}{c_{l}}\right) \dot{R}^{2}=\left(1+\frac{\dot{R}}{c_{l}}\right) \frac{P_{S}}{\rho_{l}}+\frac{R}{\rho_{l} c_{l}} \frac{d P_{S}}{d t}
$$

where $R$ is the bubble radius, $c_{l}$ is the speed of sound in the surrounding liquid and $\rho_{l}$ is the liquid density. The dotted accents denote time derivatives. $P_{S}$ is given by

$$
\mathrm{P}_{\mathrm{S}}=P_{B}(t)+p_{v}-\frac{2 \sigma}{R}-\frac{4 \mu \dot{R}}{R}-P_{\infty}(t)
$$


$P_{B}$ is the pressure inside the bubble and $P_{\infty}$ is the pressure in the liquid far from the bubble centre. $\mu$ is the dynamic viscosity. $\sigma$ is the surface tension between the gas in the bubble and the liquid. $p_{v}$ is the sum of the vapor pressure and an estimation of the local values of turbulent pressure fluctuations:

$$
p_{v}=p_{\text {sat }}+0.39 \frac{\rho k}{2}
$$

where $p_{\text {sat }}$ is vapour pressure ${ }^{14}$.

\section{Flow equations}

Fluid flow is governed by the Reynolds-averaged Navier-Stoke (RANS) equations:

$$
\begin{gathered}
\frac{\partial \rho}{\partial t}+\nabla \cdot(\rho \boldsymbol{u})=0 \\
\frac{\partial\left(\rho u_{i}\right)}{\partial t}+\nabla \cdot\left(\rho \boldsymbol{u} u_{i}\right)=\nabla \cdot\left[\left(\mu+\mu_{t}\right) \nabla u_{i}\right]+S_{u_{i}}
\end{gathered}
$$

$\boldsymbol{u}$ is the fluid velocity, $\mu_{t}$ is the eddy viscosity and $S_{u_{i}}$ are the momentum sources, including buoyancy.

The standard $k-\varepsilon$ turbulence model is used for closure ${ }^{26}$ with an additional source term $S_{k}$ to account for turbulence generated due to bubble collapse.

$$
\begin{aligned}
& \frac{\partial(\rho \mathrm{k})}{\partial t}+\nabla \cdot(\rho \boldsymbol{u} \mathrm{k})=\nabla \cdot\left[\left(\mu+\frac{\mu_{t}}{\sigma_{k}}\right) \nabla \mathrm{k}\right]+\mu_{\mathrm{t}} G-\rho \varepsilon+S_{k} \\
& \frac{\partial(\rho \varepsilon)}{\partial t}+\nabla \cdot(\rho \boldsymbol{u} \varepsilon)=\nabla \cdot\left[\left(\mu+\frac{\mu_{t}}{\sigma_{\varepsilon}}\right) \nabla \varepsilon\right]+\mathrm{C}_{1 \varepsilon} \mu_{\mathrm{t}} G \frac{\varepsilon}{k}-C_{2 \varepsilon} \rho \frac{\varepsilon^{2}}{k} \mathrm{q}
\end{aligned}
$$

$G$ is the turbulence generation rate. The constants $\sigma_{k}, \sigma_{\varepsilon}, \mathrm{C}_{1 \varepsilon}$ and $\mathrm{C}_{2 \varepsilon}$ are $1.00,1.30,1.44$ and 1.92 respectively as per the original model. 
The velocity generated by bubble collapse is estimated from the Keller-Miksis equation (6), ignoring the second-order derivative of $R$ and the time derivative of the bubble

pressure, as:

$$
v_{k}=\left[\frac{2}{3}\left(\frac{1+\frac{\sqrt{k}}{c}}{1-\frac{1 \sqrt{k}}{3 c}}\right) \frac{p_{v}-p_{t o t}}{\rho_{l}}\right]^{\frac{1}{2}}
$$

where the interfacial velocity of the bubble is approximated by $\sqrt{k}$. The total pressure $p_{\text {tot }}$ is given by

$$
p_{\text {tot }}=p+p_{0}+\rho g h
$$

where $p_{0}$ is the atmospheric pressure and $h$ is the depth below the free surface. The term $\rho g h$ is significant for dense liquids like aluminium. The additional turbulent source term in the $k$ equation then becomes:

$$
S_{k}=f_{g} \rho \frac{v_{k}^{2}}{t_{c}}
$$

where $t_{c}$ is a characteristic time scale for the lifetime of the bubbles.

\section{Mass fraction transport equation}

The growth and collapse rates of the cavitation bubbles are derived using the same steps as the full cavitation model of Singhal et al. ${ }^{14}$, but using the Keller-Miksis equation instead, since this research is concerned with large forcing amplitudes. Combining the phase continuity equations expressed in terms of volume fraction $\alpha$ :

$$
\frac{\partial\left[(1-\alpha) \rho_{l}\right]}{\partial t}+\nabla \cdot\left[(1-\alpha) \rho_{l} \boldsymbol{u}\right]=\Gamma
$$




$$
\frac{\partial\left(\alpha \rho_{b}\right)}{\partial t}+\nabla \cdot\left(\alpha \rho_{b} \boldsymbol{u}\right)=-\Gamma
$$

and the continuity equation (8), and relating the volume fraction to the bubble density $\mathrm{N}$, the following relation is obtained:

$$
\frac{D \rho}{D t}=-\left(\rho_{l}-\rho_{b}\right) \sqrt[3]{4 \pi N(3 \alpha)^{2}} \frac{D R}{D t}
$$

Using the Keller-Miksis equation (6), and ignoring the viscous, surface tension terms and acceleration term, that is important only during the initial bubble acceleration, the expression for the net phase change rate $\Gamma$ is obtained as

$$
\Gamma=\sqrt[3]{4 \pi N(3 \alpha)^{2}} \frac{\rho_{l} \rho_{b}}{\rho} \sqrt{\frac{2}{3}\left(\frac{1+\frac{\sqrt{k}}{c}}{1-\frac{1 \sqrt{k}}{3 c}}\right) \frac{p_{v}-p_{t o t}}{\rho_{l}}}
$$

where the interfacial velocity of the bubble is again approximated by $\sqrt{k}$.

The use of the Keller-Miksis equation to derive the phase change source terms assumes the following:

- The internal pressure of the bubbles is homogeneous, since the inertia of the gas is negligible.

- The bubbles remain spherical. Due to the large value of the surface tension of hydrogen interfaces with liquid aluminium, bubbles observed in melt cavitation treatment are generally small, in the region of $10-100 \mu \mathrm{m}$ in radius ${ }^{27}$.

Expressing phase change in terms of mass fractions, the mass fraction transport equation is given by:

$$
\frac{\partial\left(\rho_{g} f_{g}\right)}{\partial t}+\nabla \cdot\left(\rho_{g} \boldsymbol{u} f_{g}\right)=\nabla \cdot\left(\Gamma \nabla f_{g}\right)+\Gamma_{G}-\Gamma_{C}
$$


where $\Gamma_{G}$ and $\Gamma_{C}$ are the growth and collapse source terms respectively and $\Gamma=\Gamma_{G}-\Gamma_{C}$. The interfacial velocity of the bubble is approximated as $\sqrt{k}$, giving:

when $p<p_{v}$ :

$\Gamma_{G}=C_{G} \frac{\sqrt{k}}{\sigma} \rho_{l} \rho_{g}\left[\frac{2}{3}\left(\frac{1+\frac{\sqrt{k}}{c}}{1-\frac{1 \sqrt{k}}{3 c}}\right) \frac{p_{v}-p_{t o t}}{\rho_{l}}\right]^{\frac{1}{2}}\left(1-f_{g}\right)$

when $p>p_{v}$ :

$$
\Gamma_{C}=C_{c} \frac{\sqrt{k}}{\sigma} \rho_{l} \rho_{g}\left[\frac{2}{3}\left(\frac{1+\frac{\sqrt{k}}{c}}{1-\frac{1 \sqrt{k}}{3 c}}\right) \frac{p_{t o t}-p_{v}}{\rho_{l}}\right]^{\frac{1}{2}}\left(f_{g}\right)
$$

where $C_{G}=0.02$ and $C_{C}=0.01$. In the absence of known values for $N$, equation (18) has been re-expressed in terms of the bubble radius, and invoking the relation ${ }^{28}$

$$
\mathrm{R}=\frac{0.061 W e \sigma}{2 \rho_{l} k}
$$

to express the source terms in terms of known quantities.

\section{Algorithm}

\section{Wave equation solution}

The wave equations (2) and (3) are initially solved using the leapfrog scheme, implemented in Greenwich's in-house multiphysics package PHYSICA ${ }^{29}$, with a time-step of $1 \mu$ s for a simulation time of $1 \mathrm{~ms}$. In the leapfrog scheme, the pressures are stored at cell centres at each time-step. Velocity components of the pressure perturbations are stored on faces half a time-step apart from the pressures. The values for pressure and velocity components at the next iteration are given by: 


$$
\begin{aligned}
& p^{n}=p^{n-1}-\frac{c \Delta t}{x_{i}}\left(u_{i, d w}^{n-1 / 2}-u_{i, u p}^{n-1 / 2}\right)+S_{m a s s} \Delta t \\
& u_{i}^{n+1 / 2}=u_{i}^{n-1 / 2}-c \Delta t\left(p_{d w}^{n}-p_{u p}^{n}\right)+S_{i} \Delta t
\end{aligned}
$$

where $n$ is the iteration number and $x_{i}$ is the cell distance in the ith direction. The subscripts $d w$ and up denote the downwind and upwind values respectively.

The iterative procedure in each time-step is as follows:

(1) The mixture density and speed of sound are updated using equations (4) and (5).

(2) The acoustic pressure is solved using the discretized form of equation (2).

(3) The velocities are then solved using the discretized form of equation (3).

(4) The mass fraction transport equation (19) is solved.

(5) The minimum and maximum acoustic pressures are stored in each cell.

\section{Averaged homogeneous cavitation model solution}

The cavitation model is then run for a longer simulation time of $20 \mathrm{~s}$, with a larger timestep of $1 \mathrm{~ms}$. The transport equations are discretized using the finite volume method in a segregated manner ${ }^{29}$. The evaluated local minimum and maximum acoustic pressures from the previous calculation are used in the source terms $\quad$ (21) and $\quad$ (22) respectively in this longer run: this corresponds to evaluating an average bubble growth rate in each cell. The appeal of using two calculations with different time scales lies in the reduced run time from weeks to hours, by enabling the use of large time-steps for cavitating flow simulations.

\section{Problem Description}

In order to model the continuous treatment of liquid with ultrasound, a launder geometry as shown in Figure 1 is used for the computational domain. The sonotrode is located at the centre of 
the domain, equidistant between the two baffles. The horn is immersed $1 \mathrm{~cm}$ below the liquid surface. The frequency of the sonotrode is $20 \mathrm{kHz}$ and the amplitude of the acoustic pressure below the horn is 5.0 MPa. This amplitude is at the upper limit of acoustic pressures that can be encountered during melt treatment experiments: Xu et al. ${ }^{27}$ used acoustic pressures of the order of 4.0 MPa in their experiments.

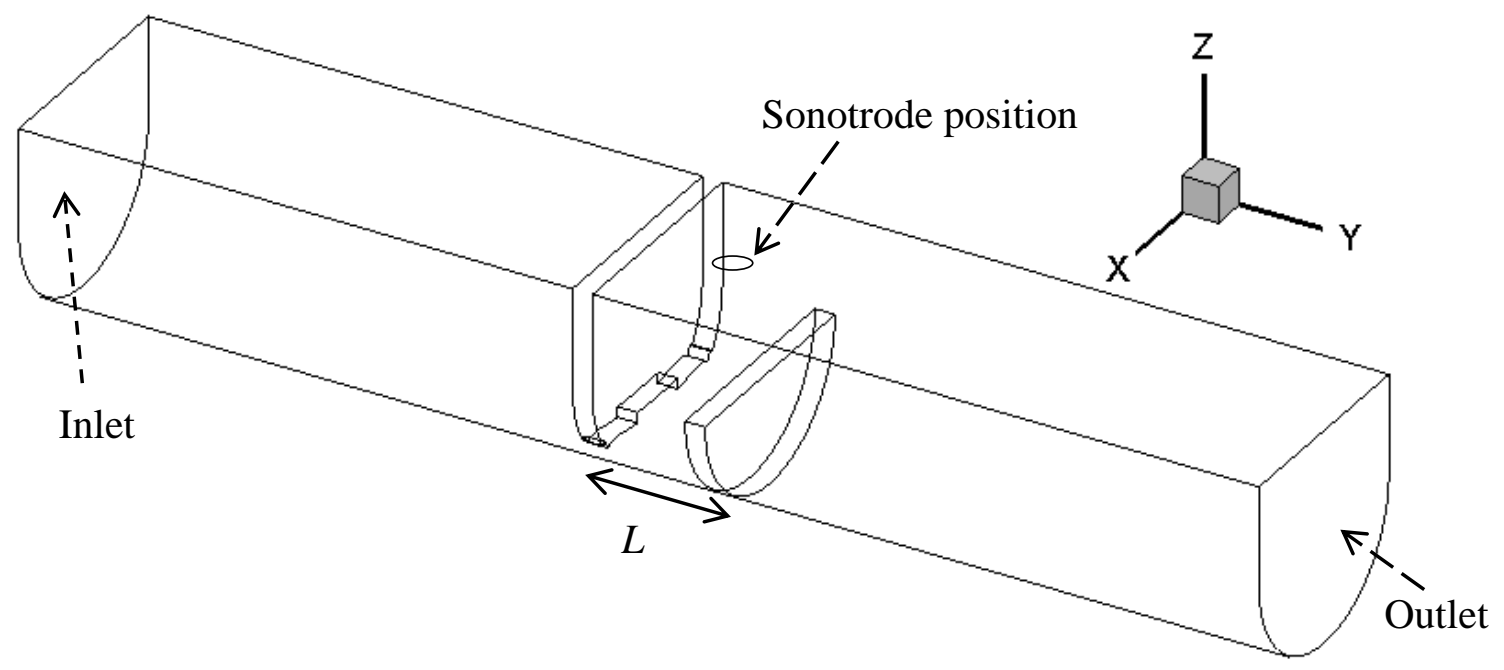

$150 \mathrm{~cm} \times 9 \mathrm{~cm} \times 8 \mathrm{~cm}$ launder. L denotes the length between baffles, of thickness $8 \mathrm{~mm}$ each. The sonotrode is immersed $1 \mathrm{~cm}$ into the free surface at the centre of the domain.

The inlet and outlet are at the low and high y boundaries respectively. The liquid enters the domain at a velocity of $0.01 \mathrm{~m} \mathrm{~s}-1$ in the y direction. The launder problem is solved for both water at $20^{\circ} \mathrm{C}$ and aluminium at $700{ }^{\circ} \mathrm{C}$; material properties for both water and aluminium are given in Table 1. The launder boundaries and the liquid free surface are assumed to be fully reflective to sound waves. A phase shift of $180^{\circ}$ occurs when pressure waves reflect from the free surface: this is implemented by setting the acoustic pressures in the computational cells located just above the free surface to $0 \mathrm{~Pa}$. 
The distance between the baffles is a parameter that is varied as a function of the sound wavelength in the travelling medium. In water, the wavelength $\lambda$ of ultrasound at $20 \mathrm{kHz}$ is 7.4 $\mathrm{cm}$, and in aluminium, the wavelength is $23 \mathrm{~cm}$. The simulation is run for each liquid for the following distances $L$ between baffles: $0.5 \lambda, 1.0 \lambda$ and $1.5 \lambda$ using the procedure described in the theory section.

Mesh independence is determined by investigating the predicted pressure differences in the domain when using meshes with 8770, 16768, 22518 and 30486 cells as shown in Figures 2 and 3. The mesh with 30486 cells is used for computing the results presented in the next section.

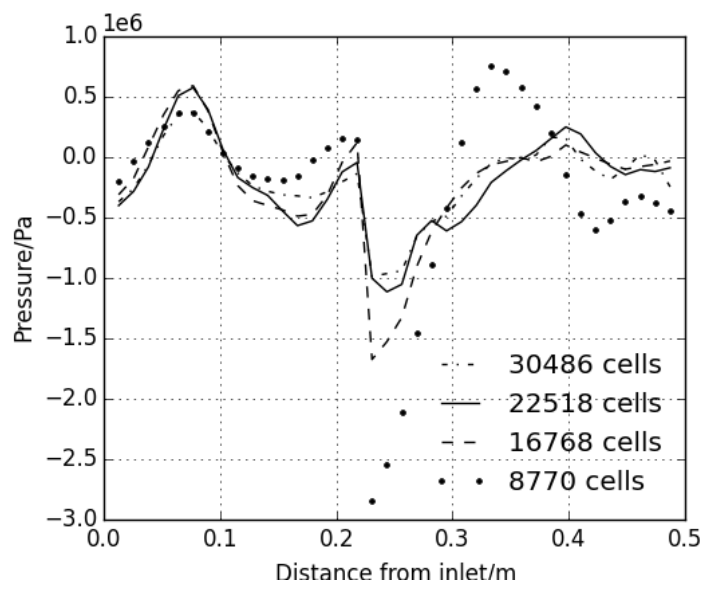

2 Predicted acoustic pressure along axis of launder with different meshes

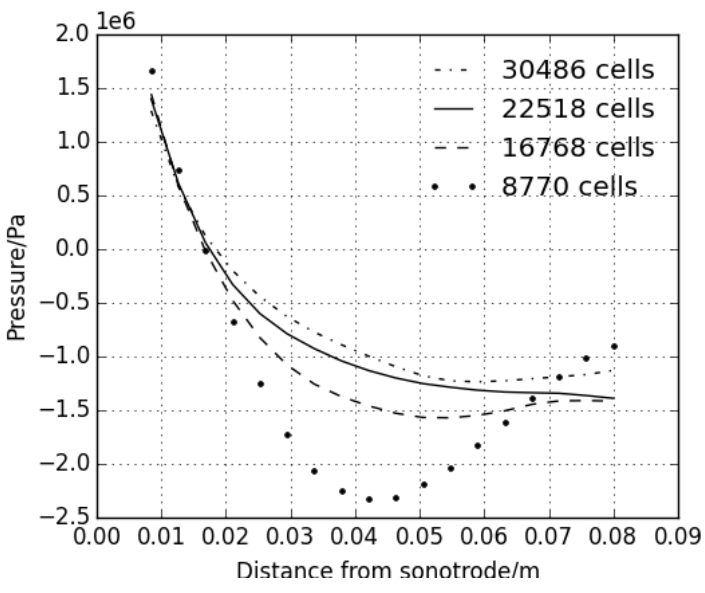

3 Predicted acoustic pressure along axis of sonotrode with different meshes

The large variations in the acoustic pressures with different mesh sizes are due to the sensitivity of cavitation models to the location and number of initial bubble sizes. In the homogeneous cavitation model presented in this paper, the choice of fixed finite volume cells imposes local length scales (of the order of the cube root of the cell volume) on the problem: processes occurring at smaller scales cannot be resolved ${ }^{30}$. The model therefore predicts the average behaviour of a mixture (of liquid and bubbles) in each cell, instead of resolving the dynamics of each individual bubble. When changing the grid size, the computed cell densities 
and speeds of sound change, thereby having a knock-on effect on subsequent computations of acoustic pressures. This is an inherent trait of cavitation modelling: addressing this feature will necessitate uncertainty quantification modelling of the cavitation process, with many simulations run with slightly different parameters and gauging the effect of small changes in material properties, initial and boundary conditions, and mesh size on the macroscopic flow and pressure pattern. This will be the subject of future studies. The speed-up offered by the time-averaging algorithm presented in this paper makes such a systematic study affordable.

\section{Results}

\section{Cavitation of a $100 \mu \mathrm{m}$ bubble}

Cavitating bubbles with radii of the order of $100 \mu \mathrm{m}$ have been observed in X-ray imaging of Al$10 \mathrm{Cu}$ samples ${ }^{27}$. Solving the Keller-Miksis equation using the standard Runge-Kutta method for bubbles of this size in water and aluminium, for a forcing sinusoidal signal of amplitude 5.0 MPa and frequency $20 \mathrm{kHz}$, results in the radius evolution profiles depicted in Figures 4 and 5 respectively. For both materials, bubble collapses occurs at $0.1 \mathrm{~ms}$ intervals: the characteristic time-scale $t_{c}$ used in term (15) is therefore assumed to be $0.1 \mathrm{~ms}$.

Table 1 Material properties of liquid aluminium and water

\begin{tabular}{|l|l|l|}
\hline Material Property & Aluminium $\left(700^{\circ} \mathrm{C}\right)$ & Water $\left(20^{\circ} \mathrm{C}\right)$ \\
\hline Sound speed $c(\mathrm{~m} \mathrm{~s}-1)$ & 4600 & 1481 \\
Density $\rho_{l}(\mathrm{~kg} \mathrm{~m}-3)$ & 2350 & 1000 \\
Bulk modulus $K(\mathrm{GPa})$ & 41.2 & 2.15 \\
Dynamic viscosity $\mu(\mathrm{mPa} \mathrm{s})$ & 1.3 & 0.798
\end{tabular}




\begin{tabular}{|l|l|l|} 
Surface tension (hydrogen interface) $S(\mathrm{~N} \mathrm{~m}-1)$ & 0.87 & 0.072 \\
Vapour pressure $p_{\text {sat }}(\mathrm{kPa})$ & 0 & 4.24 \\
\hline
\end{tabular}

Improved formulation for the radius evolution will include contributions from the Bjerknes forces, as is the case for microscopic models ${ }^{31}$ : this is the subject of current investigation and will be detailed in future publications.

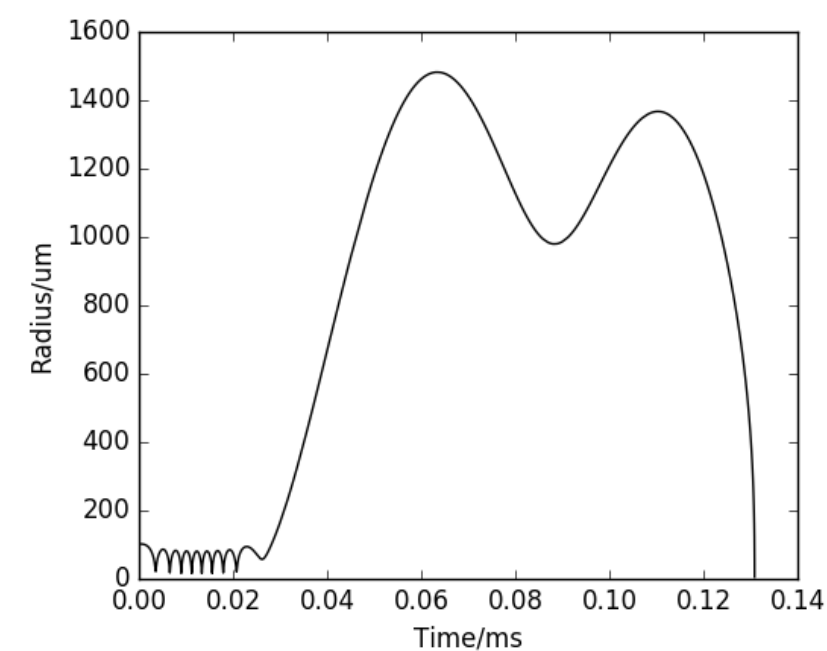

4 Radius evolution for a bubble of initial radius $100 \mu \mathrm{m}$ in water

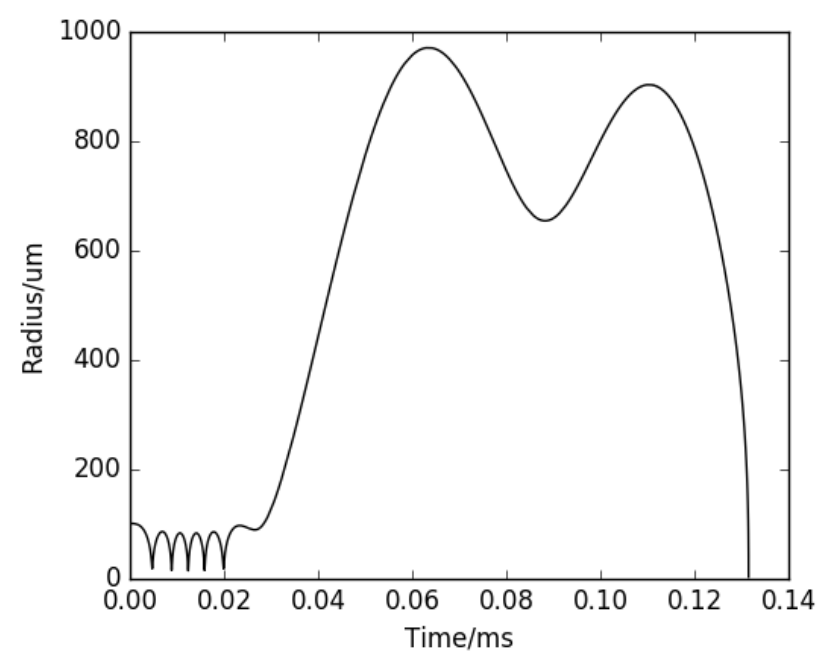

5 Radius evolution for a bubble of initial radius $100 \mu \mathrm{m}$ in aluminium

\section{Acoustic pressure solution}

Solving the wave equations (2) and (3) gives the minimum and maximum acoustic pressure contours across the whole domain. The minimum acoustic pressure contour plots along the central $\mathrm{x}$ and $\mathrm{y}$ planes are shown in Figures 6 and 7. As expected, the extreme pressure values are found below the sonotrode, where the cavitation activity is expected to be most intense, inside the conical bubble structure. The cavitation threshold of $-100.0 \mathrm{kPa}$ (acoustic pressure) in water is achieved everywhere in the domain. 


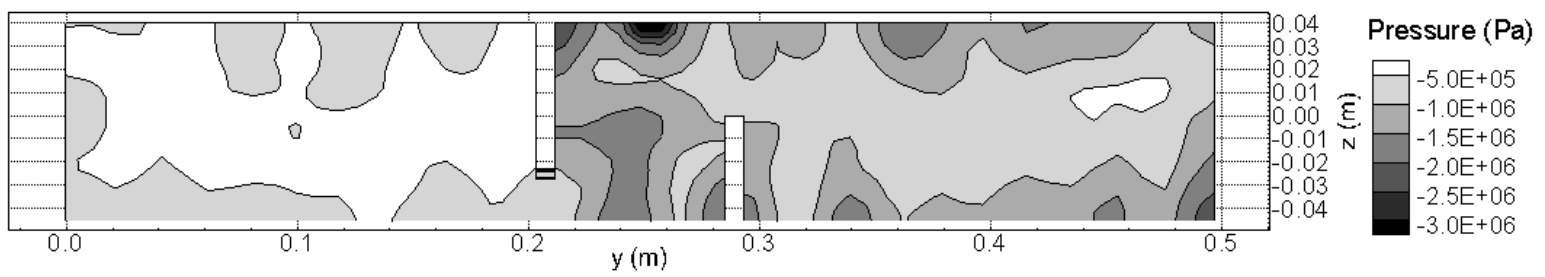

6 Predicted minimum instantaneous acoustic pressure $(\mathrm{Pa})$ in domain for a configuration with baffles separated at a distance of $1.0 \lambda$ for water along the central x plane

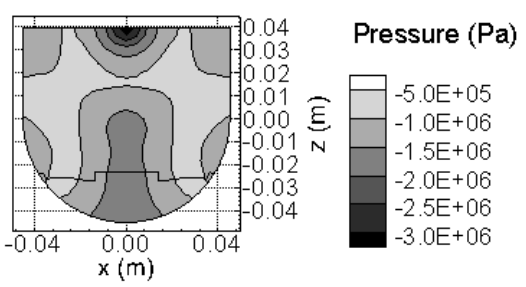

7 Predicted minimum instantaneous acoustic pressure $(\mathrm{Pa})$ in domain for a configuration with baffles separated at a distance of $1.0 \lambda$ for water along the central y plane

\section{Cavitation run solution}

The minimum and maximum pressure fields from the wave equation solution are used in the source terms of the bubble transport equation (20). The resulting bubble mass fraction plots along the axes of the domain are shown in Figures 8, 9, 10 and 11. These results are obtained in a run time of the order of 1.5 days on a $3.60 \mathrm{GHz} \mathrm{CPU}$ on the converged mesh of 30486 cells, making this approach attractive for industrial and optimization applications.

For both water and aluminium, a baffle separation of $1.0 \lambda$ maximizes the bubble concentration in the domain. The flow generated with this baffle distance also convects the maximum concentration of bubbles downstream, resulting in a larger number of nucleation sites at the outlet. The baffle distances at half integers of wavelength are equally poor at yielding a large bubble concentration downstream. A larger bubble concentration is desired, since higher cavitation activity - occurring as more bubbles collapse - is thought to promote better grain 
refinement. The random cavitation of bubbles, that emit high pressure shock waves, disrupt the solidification front advancement, thus refining the grain structure ${ }^{1}$.

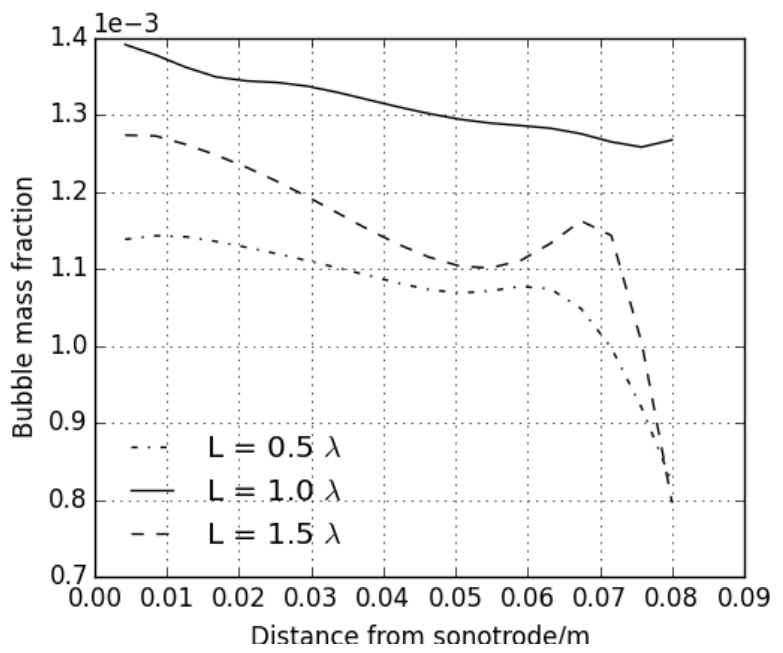

8 Bubble mass fraction along the axis of the sonotrode for water after a run time of $20 \mathrm{~s}$. Mass fraction values are taken along the axis of the sonotrode

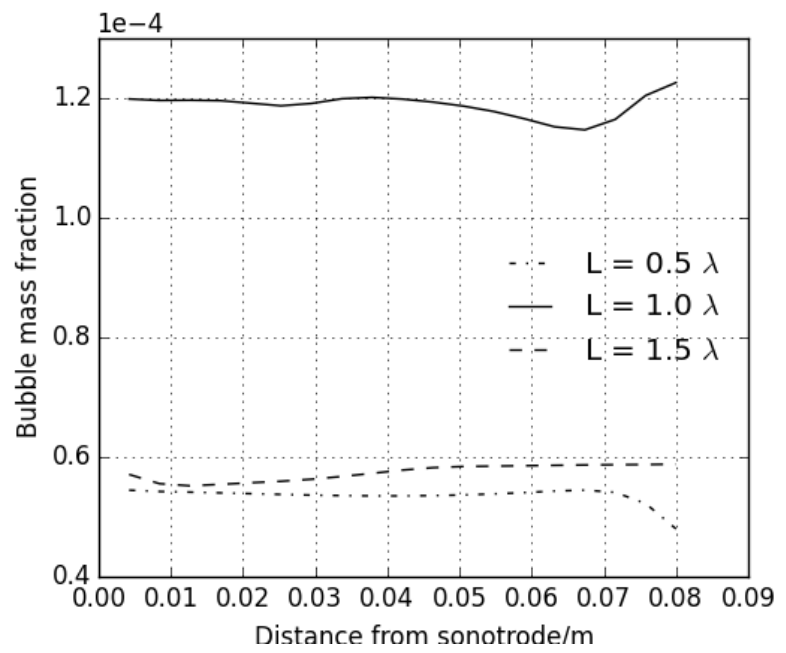

10 Bubble mass fraction along the axis of the sonotrode for aluminium after a run time of $20 \mathrm{~s}$. Mass fraction values are taken along the axis of the sonotrode

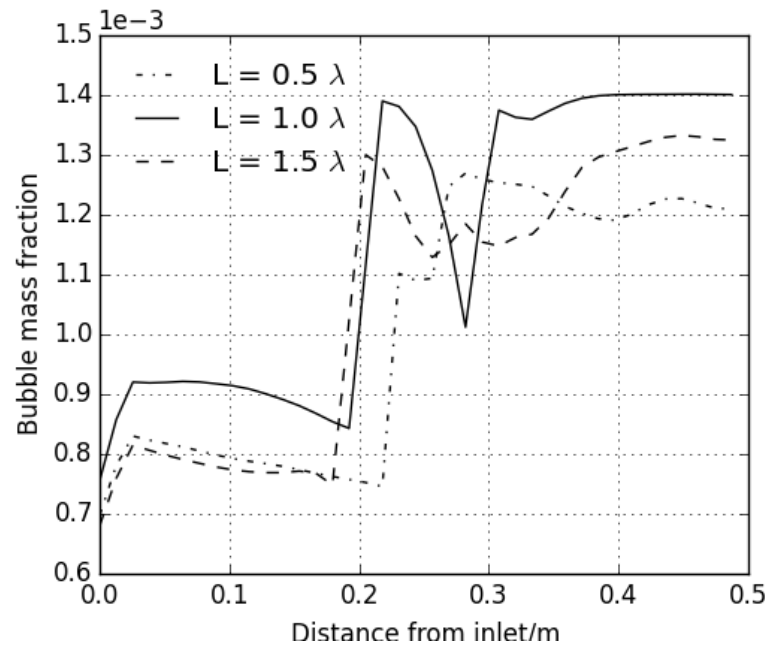

9 Bubble mass fraction across the launder for water after a run time of $20 \mathrm{~s}$. Mass fraction values are taken along the axis of the launder

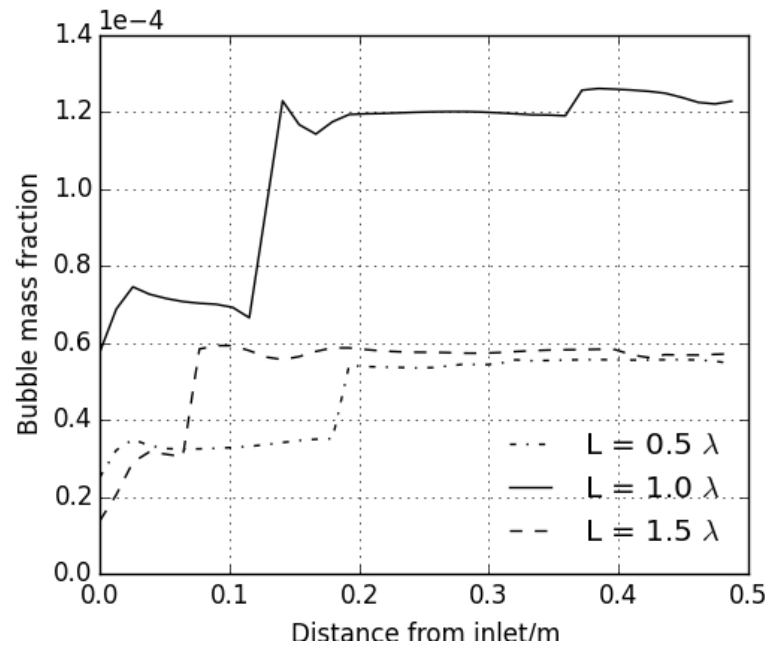

11 Bubble mass fraction across the launder for aluminium after a run time of $20 \mathrm{~s}$. Mass fraction values are taken along the axis of the launder 
Observing the flow field, two recirculation patterns are seen in two regions: under the sonotrode and downstream of the second baffle. The recirculation pattern is depicted for the aluminium case with a baffle separation of $0.5 \lambda$ in Figure 12. The second recirculation pattern can be exploited by adding a second sonotrode above this region.

The minimum residence time, the time taken for the liquid to leave the domain along the shortest path, is given in Table 2. These values are dependent on the flow pattern that is altered due to the baffle separation and the change in local densities due to cavitation activity. The wavelength of aluminium is large compared with the size of the modelled launder, giving the unusually short time to leave the domain for a separation of $1.5 \lambda$ since the baffles are close to the inlet and outlets.

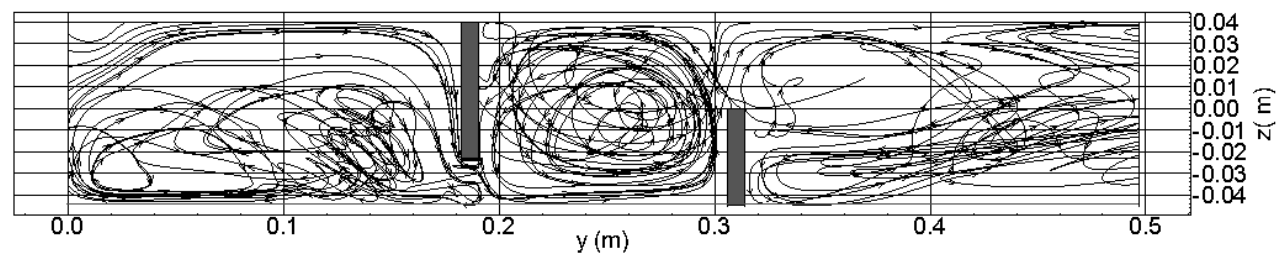

Figure 12 Recirculation in configuration with baffles separated at a distance of $0.5 \lambda$ for aluminium

Table 2 Minimum residence time (s) for each case

\begin{tabular}{|l|l|l|l|l|}
\hline L $(\lambda)$ & Laluminium $(\mathrm{cm})$ & Aluminium $(\mathrm{s})$ & Lwater $_{\text {(cm) }}$ & Water $(\mathrm{s})$ \\
\hline 0.5 & 11.5 & 7.0 & 3.7 & 2.6 \\
1.0 & 23.0 & 7.3 & 7.4 & 1.2 \\
1.5 & 34.5 & 0.2 & 11.1 & 2.4 \\
\hline
\end{tabular}




\section{Conclusions}

A multiscale model for the ultrasonic treatment of liquid metals is presented in this study. The bubble concentration for the flow in a launder with baffles to create recirculation zones in high cavitation activity regions is studied with an improved version of the full cavitation model derived from the Keller-Miksis equation.

The optimal baffle distance for both liquid water and aluminium has been found to be at one wavelength of the forcing frequency, implying that resonance with the driving frequency is a desired trait for ultrasonic cavitation treatment of liquid metal.

\section{Acknowledgement}

The authors are grateful to the UK Engineering and Physical Sciences Research Council (EPSRC) for financial assistance for this research in contract numbers: EP/K00588X/1 and EP/K005804/1.

\section{ORCID}

G S Bruno Lebon http://orcid.org/0000-0002-9389-1329

Iakovos Tzanakis http://orcid.org/0000-0002-8258-1034

Koulis Pericleous http://orcid.org/0000-0002-7426-9999

Dmitry Eskin http://orcid.org/0000-0002-0303-2249

\section{References}

1. G. I. Eskin and D. G. Eskin: 'Ultrasonic Treatment of Light Alloy Melts'; 2014, Boca Raton, FL, USA, CRC Press.

2. J. Campbell, International Metals Reviews, 1981, 26(1), 71-108.

3. O. V. Abramov, Ultrasonics, 1987, 25(2), 73-82.

4. N. Alba-Baena and D. Eskin: 'Kinetics of ultrasonic degassing of aluminum alloys', in 'Light Metals 2013', (ed. B. A. Sadler), 957-962; 2013, Hoboken, NJ, USA, John Wiley \& Sons, Inc.

5. G. I. Eskin, Ultrasonics Sonochemistry, 1995, 2(2), S137-S141.

6. Y. C. Wang, Proceedings ASME Symposium on Cavitation and Gas-Liquid Flows in Fluid Machinery and Devices, 1995, 226, 17-29.

7. E. Neppiras, Ultrasonics, 1984, 22(1), 25-28.

8. S. Komarov, K. Oda, Y. Ishiwata, and N. Dezhkunov, Ultrasonics Sonochemistry, 2013, 20(2), 754-761. 
9. I. Senocak and W. Shyy: 'Evaluation of cavitation models for Navier-Stokes computations', ASME 2002 Joint US-European Fluids Engineering Division Conference, 2002, 395-401.

10. I. Senocak and W. Shyy, Journal of Computational Physics, 2002, 176(2), 363-383.

11. C. L. Merkle, J. Feng, and P. E. O. Buelow: 'Computational modeling of the dynamics of sheet cavitation', 3rd International Symposium on Cavitation, Grenoble, France, 1998, 47-54.

12. V. Ahuja, A. Hosangadi, and S. Arunajatesan, Journal of Fluids Engineering, 2001, 123(2), 331-340.

13. R. F. Kunz, D. A. Boger, D. R. Stinebring, T. S. Chyczewski, J. W. Lindau, H. J. Gibeling, S. Venkateswaran, and T. Govindan, Computers \& Fluids, 2000, 29(8), 849875.

14. A. K. Singhal, M. M. Athavale, H. Li, and Y. Jiang, Journal of Fluids Engineering, 2002, 124(3), 617-624.

15. M. M. Athavale, H. Y. L. Yu Jiang, and A. K. Singhal, International Journal of Rotating Machinery, 2002, 8(1), 45-56.

16. J. Sauer and G. H. Schnerr, ZAMM - Journal of Applied Mathematics and Mechanics / Zeitschrift für Angewandte Mathematik und Mechanik, 2001, 81(S3), 561-562.

17. M. S. Plesset, J. Appl. Mech., 1949, 16, 277-282.

18. M. S. Plesset and A. Prosperetti, Annual Review of Fluid Mechanics, 1977, 9, 145-185.

19. C. E. Brennen: 'Cavitation and bubble dynamics'; 1995, New York, USA, Oxford University Press.

20. J.-P. Franc and J.-M. Michel: 'Fundamentals Of Cavitation'; 2004, Dordrecht, Kluwer Academic Publishers.

21. L. Nastac, Metallurgical and Materials Transactions B, 2011, 42(6), 1297-1305.

22. J. B. Keller and M. Miksis, The Journal of the Acoustical Society of America, 1980, 68(2), 628-633.

23. G. S. B. Lebon, I. Tzanakis, K. A. Pericleous, and D. G. Eskin, Journal of Physics: Conference Series, 2015, 656(1), 012134.

24. J. J. Jasper, Journal of physical and chemical reference data, 1972, 1(4), 841-1010.

25. Y.-J. Chen, W.-N. Hsu, and J.-R. Shih, Materials Transactions, 2009, 50(2), 401-408.

26. B. E. Launder and D. Spalding, Computer methods in applied mechanics and engineering, 1974, 3(2), 269-289.

27. W. W. Xu, I. Tzanakis, P. Srirangam, S. Terzi, W. U. Mirihanage, D. G. Eskin, R. H. Mathiesen, A. P. Horsfield, and P. D. Lee: 'In Situ Synchrotron Radiography of Ultrasound Cavitation in a Molten Al-10Cu Alloy', in 'TMS2015 Supplemental Proceedings', 61-66; 2015, Hoboken, NJ, USA, John Wiley \& Sons, Inc.

28. N. C. Markatos and A. K. Singhal, Advances in Engineering Software (1978), 1982, 4(3), 99-106.

29. N. Croft, K. Pericleous, and M. Cross, Numerical Methods in Laminar and Turbulent Flows, 1995, 9(2), 1269-1280.

30. G. H. Schnerr, I. H. Sezal, and S. J. Schmidt, Physics of Fluids (1994-present), 2008, 20(4), 040703.

31. G. S. B. Lebon, K. Pericleous, I. Tzanakis, and D. G. Eskin, Physical Review E Statistical, Nonlinear, and Soft Matter Physics, 2015, 92(4), 043004. 